\title{
An omega-3 polyunsaturated fatty acid concentrate administered for one year decreased triglycerides in simvastatin treated patients with coronary heart disease and persisting hypertriglyceridaemia
}

\author{
P N Durrington, D Bhatnagar, M I Mackness, J Morgan, K Julier, M A Khan, M France
}

\begin{abstract}
Background-Omega-3 fatty acids, such as those present in fish oil, have been reported to prolong life in myocardial infarction survivors. These fatty acids can decrease serum triglyceride concentrations, but so far the doses used in trials examining their effects on coronary end points have had only minimal triglyceride lowering effects.

Objective-To examine the triglyceride lowering effectiveness, safety, and tolerability of Omacor, a concentrate of omega-3, long chain, polyunsaturated fatty acids from fish oil ( $84 \%$ of the total as opposed to an average of $35 \%$ in fish oil) over one year in patients with established coronary heart disease (CHD) and persisting hypertriglyceridaemia, despite receiving simvastatin in doses similar to those employed in the Scandinavian simvastatin survival study.

Subjects and methods-59 patients with CHD, receiving simvastatin 10-40 mg daily with serum triglycerides $>2.3 \mathrm{mmol} / \mathrm{l}$, were randomised to receive Omacor $2 \mathrm{~g}$ twice a day or placebo for 24 weeks in a double blind trial. Forty six patients accepted the offer of active treatment for a further 24 weeks in an open phase of the trial.

Results-There was a sustained significant decrease in serum triglycerides by $20-30 \%$ $(\mathrm{p}<0.005)$ and in very low density lipoprotein (VLDL) cholesterol by $30-40 \%(\mathrm{p}<0.005)$ in patients receiving active Omacor at three, six, and 12 months compared either to baseline or placebo. Omacor did not have any deleterious effect on low density or high density lipoprotein cholesterol or on biochemical and haematological safety tests. There was no adverse effect on glycaemic control in patients with diabetes, who showed a decrease in serum triglyceride, which was at least as great as in non-diabetic patients. One patient receiving placebo died of acute myocardial infarction. Three patients withdrew from the trial (two on placebo and one on active treatment). Omacor was generally well tolerated.

Conclusion-Omacor was found to be a safe and effective means of lowering serum triglycerides over one year in patients with CHD and combined hyperlipidaemia, whose triglycerides remained elevated despite simvastatin treatment.

(Heart 2001;85:544-548)
\end{abstract}

Keywords: coronary heart disease; hypertriglyceridaemia; polyunsaturated fat; statin treatment

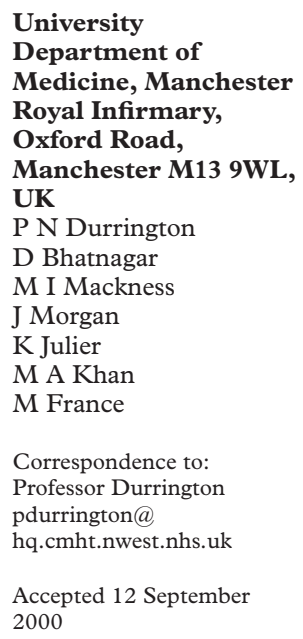

University

epartment of

Royal Infirmary,

Oxford Road,

UK

P N Durringto

K Julier

M A Khan

Correspondence to:

Professor Durrington pdurrington@

Accepted 12 September
There is strong evidence that statin treatment to decrease low density lipoprotein (LDL) cholesterol decreases mortality in patients with coronary heart disease (CHD). ${ }^{1-3}$ Frequently such patients also have hypertriglyceridaemia, which may persist after treatment with statins, and it is not known whether they could benefit from additional treatment to correct this. Omega-3 polyunsaturated fatty acids (PUFA) lower serum triglycerides ${ }^{4}$ and short term studies suggest that they also do so in patients receiving statins. $^{5-7}$. There are now at least three clinical trials to suggest that omega-3 PUFA in the diet or taken as a supplement decrease coronary mortality in established CHD. ${ }^{8-10}$ However, in none of these was the quantity of omega-3 PUFA received by the participants sufficient to lower serum triglycerides substantially. Clinicians may in future wish to employ omega-3 fatty acids in doses which lower serum triglycerides in patients with persisting hypertriglyceridaemia despite statin treatment. Omega-3 fatty acids in these higher doses might thus form the basis of a future clinical event trial.
The present trial set out to establish whether the effect of omega-3 fatty acids, in doses which substantially lower elevated serum triglycerides in the short term, was maintained over one year and whether there were any adverse effects resulting from their combination with simvastatin in these doses. The fatty acids in fish oil, which are active in lowering serum triglycerides, are the long chain highly polyunsaturated ones, principally eicosapentaenoate and docosahexaenoate, which comprise only around $35 \%$ of the fatty acids present in fish oil. A more concentrated preparation of omega-3 PUFA, Omacor, was used in this study, because an earlier short term trial suggested that the smaller dose of oil and the omission of the other fatty acids might avoid some unwanted effects. ${ }^{11}$

\section{Methods}

PATIENTS AND TRIAL DESIGN

Fifty nine consecutive, eligible patients (43 men) aged $\leqslant 75$ years (mean age 55 years, range 37-75 years), all of whom had established CHD and were attending lipid clinics at 
Table 1 Baseline characteristics (mean (SD)) of patients who entered the trial, randomised to receive omega-3 PUFA (Omacor) or placebo

\begin{tabular}{lll}
\hline & $\begin{array}{l}\text { Omega-3 PUFA } \\
\text { concentrate }\end{array}$ & Placebo \\
\hline Patients (n) (n) & 30 & 29 \\
Men/women (n) & $20 / 9$ \\
Diabetes mellitus (n) & $23 / 7$ & 7 \\
Simvastatin 40 mg/daily (n) & 8 & 10 \\
Simvastatin 20 mg/daily (n) & 10 & 17 \\
Simvastatin 10 mg/daily (n) & 20 & 2 \\
Age (years) & 0 & $54.8(10.2)$ \\
Body mass index (kg/m $\left.{ }^{2}\right)$ & $55.2(7.0)$ & $28.4(4.2)$ \\
Fasting glucose (all patients) (mmol/l) & $28.8(2.8)$ & $6.7(2.7)$ \\
Fasting glucose (diabetic patients) (mmol/1) & $6.7(2.5)$ & $10.8(2.4)$ \\
HbA $_{1 \mathrm{c}}$ (all patients) (\%) & $9.8(2.6)$ & $5.4(1.5)$ \\
$\mathrm{HbA}_{1 \mathrm{c}}$ (diabetic patients) (\%) & $5.9(1.4)$ & $7.6(1.3)$ \\
Plasma fibrinogen (g/l) $_{\text {Serum phospholipid eicosapentaenoic acid (mg/l) }}$ & $6.7(1.9)$ & $3.4(1.4)$ \\
Serum phospholipid docosapentaenoic acid (mg/l) & $72.6(1.9)$ & $21.0(12.7)$ \\
& $72.9(24.5)$ & $75.4(25.6)$ \\
\hline
\end{tabular}

the Manchester Royal Infirmary and the Royal Oldham Hospital, participated. All of them gave their informed consent to take part in the trial which was approved by the ethical committees of both hospitals. All had serum triglycerides exceeding $2.3 \mathrm{mmol} / \mathrm{l}$ and this persisted at randomisation. They were already being treated with simvastatin at a dose of $10-40 \mathrm{mg}$ (as used in the Scandinavian simvastatin survival study $\left.(4 S)^{1}\right)$, which had been unchanged for at least three months previously, with the aim of lowering serum cholesterol to $<5.5 \mathrm{mmol} / \mathrm{l}$. Some had not achieved this target even on $40 \mathrm{mg}$ daily. The average doses of simvastatin were $33.3 \mathrm{mg}$ daily in the active treatment group and $31.0 \mathrm{mg}$ daily in the placebo (corn oil) group, which are similar to the average dose of $28 \mathrm{mg}$ daily in $4 \mathrm{~S}^{1}{ }^{1}$ Throughout the trial the dose of simvastatin was kept constant. No patient had ever received probucol or any other lipid lowering drug except simvastatin within six weeks of commencement. Patients were randomised to receive Omacor, a concentrated preparation of omega-3 PUFA (44\% eicosapentaenoate and $36 \%$ docosahexaenoate) in a dose of $2 \mathrm{~g}$ twice daily or corn oil in identical capsules, as placebo, for 24 weeks in a double blind trial, after which both groups were invited to receive Omacor for a further 24 weeks. Patients and investigators remained blinded to the earlier treatment groups and to all laboratory results except creatine kinase, liver function tests, and blood counts until the last patient had completed the full evaluation period of 48 weeks. CHD was defined as in the $4 \mathrm{~S}$ trial - namely, as myocardial infarction at least six months previously with definite ECG and enzyme changes or as angina with a positive exercise ECG. ${ }^{1}$ All the patients had received American Heart Association step 1 dietary advice at least six months previously and were instructed to maintain their diet unchanged throughout the trial. Fifteen patients had noninsulin dependent diabetes mellitus. Use of aspirin, $\beta$ blockers, angiotensin converting enzyme (ACE) inhibitors, calcium channel antagonists, and oral hypoglycaemic agents was similar in the placebo and Omacor groups. Any patients who had suffered a myocardial infarction within the previous six months were excluded. Blood pressure was measured to within $2 \mathrm{~mm} \mathrm{Hg}$, with the patient semirecumbent after resting for five minutes, using a mercury sphygmomanometer.

\section{LABORATORY METHODS}

Cholesterol and triglycerides were measured after an overnight fast by the CHOD-PAP method and the GPO-PAP method, respectively (both supplied by Roche Diagnostics, Lewes, UK). The coefficients of variation were $1.3 \%$ for cholesterol and $2.1 \%$ for triglyceride. Serum phospholipid fatty acids were measured by gas chromatography. Plasma was used for the isolation of lipoproteins. Very low density lipoprotein (VLDL) was isolated by ultracentrifugation at $135000 \mathrm{~g}$ for 24 hours at a density of $1.006 \mathrm{~g} / \mathrm{ml}$, using a Beckman L8-55M ultracentrifuge fitted with a Beckman 50.4 Ti Rotor (Beckman Instruments, Palo Alto, California, USA). Following ultracentrifugation the upper VLDL layer was removed for analysis of cholesterol concentration. LDL in the remaining (lower) layer was precipitated with heparin $/ \mathrm{Mn}^{2+}$ and the high density lipoprotein (HDL) cholesterol concentration in the supernatant determined. The LDL cholesterol concentration was calculated as the difference in cholesterol in the post-VLDL infranatant minus HDL cholesterol. Serum Lp(a) lipoprotein concentration was determined using an IRMA (Mercodia, Uppsala, Sweden) with a coefficient of variation of $6 \%$. Standards and controls were supplied by the manufacturer. Apolipoprotein (apo) A1 and apo B were determined by rate nephelometry on the Beckman Array (coefficient of variation 6\%). Standards and controls were supplied by the manufacturer. Plasma fibrinogen was measured on an automated coagulation laboratory analyser (Instrumentation Laboratories Ltd, Warrington, UK) by a thromboplastin initiated clotting technique. Blood glucose, creatine kinase, liver function tests, and other biochemistry were performed on the Hitachi 747 (Roche Diagnostics) and basic haematology on the Sysmex 9500 (Sysmex, Milton Keynes, UK). Glycated haemoglobin $\left(\mathrm{HbA}_{1 c}\right)$ was measured by high performance liquid chromatography (HPLC) using the variant analyser (Bio-Rad Laboratories Ltd., Hemel Hempstead, UK)

\section{STATISTICS}

Differences between placebo and control at 12, 24 , and 48 weeks were sought by analysis of covariance with the baseline as covariate. The planned primary end points in this analysis for which the study was statistically powered were serum triglycerides and VLDL cholesterol. Baseline parameters were also compared with results obtained at 12,24 , and 48 weeks using the Wilcoxon signed rank test for serum triglycerides and VLDL cholesterol, and Student's paired $t$ test for other variables. Because this involved multiple statistical testing the level of probability considered significant was 0.025 . 
Table 2 Serum lipoprotein lipid concentrations (mmol/l), apolipoprotein AI and B (mg/dl), Lp(a) lipoprotein (mg/dl), and blood pressure (mm Hg) in 59 patients treated with simvastatin randomised to receive omega-3 PUFA (Omacor) or placebo for 24 weeks after which 46 patients received omega-3 PUFA for a further 24 weeks

\begin{tabular}{|c|c|c|c|c|c|c|c|c|}
\hline Treatment & None & $\begin{array}{l}\text { Omega-3 PUFA } \\
\text { concentrate }\end{array}$ & $\begin{array}{l}\text { Omega-3 PUFA } \\
\text { concentrate }\end{array}$ & $\begin{array}{l}\text { Omega-3 PUFA } \\
\text { concentrate }\end{array}$ & None & Placebo & Placebo & $\begin{array}{l}\text { Omega-3 PUFA } \\
\text { concentrate }\end{array}$ \\
\hline Weeks & 0 & 12 & 24 & 48 & 0 & 12 & 24 & 48 \\
\hline $\mathrm{n}$ & 30 & 30 & 29 & 25 & 29 & 27 & 26 & 21 \\
\hline Serum triglycerides & $4.6(2.2)$ & $3.3(1.4)^{\star \star \star}$ & $3.5(1.8)^{\star \star \star}$ & $3.0(1.7)^{\star \star \star}$ & $3.8(2.2)$ & $3.9(2.0)$ & $3.9(2.5)$ & $2.9(1.9)^{\star \star \star}$ \\
\hline Serum cholesterol & $5.6(1.5)$ & $4.9(1.2)^{\star}$ & $5.0(1.2)^{\star}$ & $4.9(0.9)^{\star \star}$ & $6.2(1.4)$ & $6.3(1.5)$ & $6.4(1.5)$ & $6.0(1.4)$ \\
\hline VLDL cholesterol & $1.0(0.5)$ & $0.6(0.5)^{\star \star}$ & $0.6(0.4)^{\star \star}$ & $0.7(0.5)^{\star \star}$ & $0.9(0.6)$ & $0.8(0.6)$ & $0.8(0.6)$ & $0.6(0.6)^{\star \star}$ \\
\hline LDL cholesterol & $3.5(1.4)$ & $3.1(1.1)$ & $3.3(1.2)$ & $2.8(1.0)^{\star}$ & $4.2(1.6)$ & $4.2(1.7)$ & $4.4(1.7)$ & $3.7(1.8)$ \\
\hline HDL cholesterol & $1.1(0.4)$ & $1.2(0.4)$ & $1.0(0.3)$ & $1.2(0.4)$ & $1.1(0.4)$ & $1.2(0.5)$ & $1.3(0.4)$ & $1.3(0.5)$ \\
\hline Apolipoprotein AI & $90(14)$ & Not done & $84(13)$ & $96(20)$ & $89(15)$ & Not done & 89 (13) & $95(18)$ \\
\hline Apolipoprotein B & $96(31)$ & Not done & $95(26)$ & $93(24)$ & $110(31)$ & Not done & $114(33)$ & $108(34)$ \\
\hline $\mathrm{Lp}$ (a) lipoprotein & $\begin{array}{l}10.5 \\
(2.1-152.1)\end{array}$ & Not done & $16.2(1.9-99.1)$ & $15.5(2.1-105.4)$ & $26(6.7-178)$ & Not done & $\begin{array}{l}38.5 \\
(6.7-176)\end{array}$ & $26.6(5.9-176.4)$ \\
\hline $\begin{array}{l}\text { Systolic blood } \\
\text { pressure }\end{array}$ & $129(15)$ & Not done & $130(22)$ & $127(15)$ & $131(20)$ & Not done & $130(19)$ & $131(20)$ \\
\hline $\begin{array}{l}\text { Diastolic blood } \\
\text { pressure }\end{array}$ & $79(8)$ & Not done & $78(11)$ & $79(8)$ & $79(11)$ & Not done & $79(10)$ & $81(11)$ \\
\hline
\end{tabular}

Mean (SD) except Lp(a) lipoprotein, which is median (range).

Significantly different from their baseline values on paired testing: ${ }^{\star} \mathrm{p}<0.025 ;{ }^{\star \star} \mathrm{p}<0.005$; ${ }^{\star \star \star} \mathrm{p}<0.0005$.

Results of analysis of covariance are shown in fig 1 .

\section{Results}

Adverse events were reported by 22 patients receiving Omacor and 17 receiving placebo.
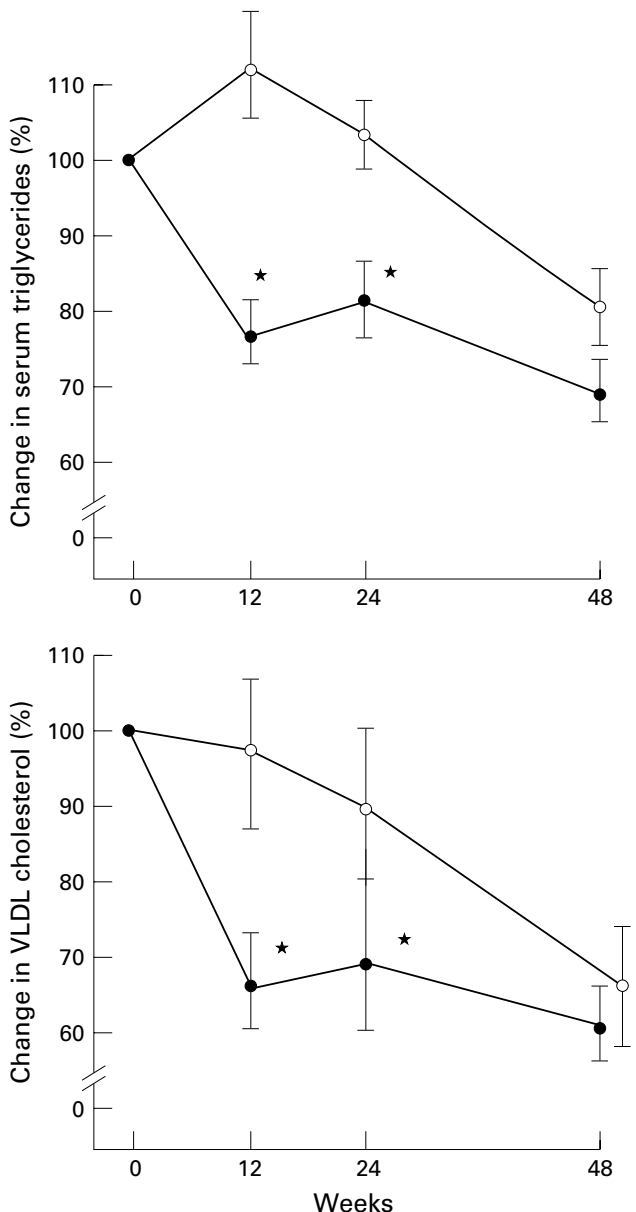

Figure 1 Change in serum triglycerides and VLDL cholesterol concentrations as a percentage of baseline values during treatment with omega-3 polyunsaturated fatty acid concentrate at a dose of $2 \mathrm{~g}$ twice a day for 48 weeks (solid circles) or with placebo for 24 weeks followed by active omega-3 polyunsaturated fatty acid concentrate from 24 to 48 weeks (open circles) in patients with coronary heart disease and serum triglycerides exceeding $2.3 \mathrm{mmol} / \mathrm{l}$ despite treatment with simvastatin ( $n=59$ at 0 weeks, 57 at 12 weeks, 55 at 24 weeks, and 46 at 24 weeks). The trial was double blind from 0-24 weeks and open from 24-48 weeks. Values are mean (SEM). *Significant with baseline concentration as covariate $(p<0.005)$.
These events were mostly minor except for one patient in the placebo group who died from acute myocardial infarction, for another patient in the placebo group who withdrew because of severe heartburn, and for one patient in the active treatment group who was withdrawn because of loose motions. Another patient receiving placebo withdrew because visits proved inconvenient. Fifty five patients thus completed the double blind, placebo controlled 24 week phase of the trial. Of these 46 agreed to take active Omacor for a further 24 weeks. The five patients who had earlier received placebo and the four who had received active treatment in the double blind phase, who declined to enter the open phase, did so because of the inconvenience of frequent hospital attendances. The results presented are for all evaluable patients at $0,12,24$, and 48 weeks. Exclusion of all patients other than the 46 present at each of these time points does not alter the conclusions. Compliance, assessed by counting returned capsules, was $>80 \%$ in all but three patients. Patients were included in the statistical analysis regardless of compliance (intention to treat analysis). Serum phospholipid eicosapentaenoate and docosahexaenoate concentrations were similar in the placebo and Omacor groups at baseline, but in patients receiving Omacor the eicosapentaenoate concentrations rose by a mean of $260 \%$ (95\% confidence interval (CI) $192 \%$ to $327 \%$ ) at six months and by $361 \%$ (95\% CI $276 \%$ to $447 \%$ ) at 12 months, and the docosahexaenoate concentrations by $54 \%$ ( $95 \%$ CI $38 \%$ to $70 \%$ ) and $52 \%(95 \%$ CI $35 \%$ to $69 \%)$ at the same time points. There were no significant changes in these parameters in patients on placebo at six months (eicosapentaenoate $12 \%$ (95\% CI $-33 \%$ to $57 \%$ ) and docosahexaenoate $-6.1 \%$ (95\% CI $-13 \%$ to $1 \%)$ ).

The patients allocated to active and placebo treatment were similar in most respects at baseline (table 1). By chance, however, the mean (SD) serum triglyceride at randomisation was $4.6(2.2) \mathrm{mmol} / \mathrm{l}$ in those allocated to the Omacor group and $3.8(2.2) \mathrm{mmol} / 1$ in those allocated to placebo. The primary end points of the trial (percentage changes in serum 
triglycerides and VLDL cholesterol) in the patients on active treatment were, however, significantly greater by the per protocol analysis of covariance than in those on placebo (fig 1 ), even after adjusting for any effect of the baseline values $(p<0.005)$. The secondary end points (concentrations of serum cholesterol, triglycerides, VLDL, and LDL cholesterol) are summarised in table 2. Serum triglyceride and VLDL cholesterol concentrations decreased significantly compared to baseline on Omacor ( $<<0.0005$ and $\mathrm{p}<0.005$, respectively). At no stage was there any tendency for LDL cholesterol to rise or for HDL cholesterol to decrease.

Changes in lipoprotein concentrations were unrelated to the dose of simvastatin, the mean decrease in serum triglycerides being $25 \%$ in those on $10-20 \mathrm{mg}$ and $27 \%$ in those on $40 \mathrm{mg}$ daily. The triglyceride, VLDL cholesterol, and serum cholesterol responses of diabetic patients to Omacor were also not significantly different from non-diabetics. Numerically they were greater, the decrease in triglyceride, for example, being $35 \%$ in the diabetic patients at the end of the double blind phase and $44 \%$ at the end of the trial. No significant change in fasting blood glucose or $\mathrm{HbA}_{1 \mathrm{c}}$ occurred in either the diabetic or non-diabetic patients receiving Omacor for 48 weeks. This was also true when the combined groups and the 15 diabetic patients receiving Omacor for 24 weeks were analysed. There were no apparent differences between placebo and Omacor in the frequency of laboratory values outside the reference range (full data available on request). No significant effects were observed on apo AI or $\mathrm{B}, \mathrm{Lp}(\mathrm{a})$ lipoprotein, fibrinogen or blood pressure.

\section{Discussion}

Omacor $2 \mathrm{~g}$ twice a day for 48 weeks decreased serum triglycerides by $20-30 \%$ and VLDL cholesterol by $30-40 \%$ in patients with combined hyperlipidaemia and CHD already receiving simvastatin $10-40 \mathrm{mg}$ daily. The patients were similar to those in the $4 \mathrm{~S}$ trial in which the statin produced a $30 \%$ decrease in mortality over five years. ${ }^{1}$ In that trial, however, patients with serum triglycerides $>2.5 \mathrm{mmol} / 1$ before the start of treatment were excluded. In two later statin trials ${ }^{23}$ patients with higher triglyceride concentrations participated. In one of these, patients with higher than average triglyceride values showed no significant reduction in CHD incidents with statin treatment. ${ }^{3}$ No trial has yet attempted to discover whether there might be additional benefit from treatment to lower triglycerides in patients already receiving a statin. Fish oil in patients with hypertriglyceridaemia has been previously reported to raise LDL cholesterol. ${ }^{12}{ }^{13}$ In the present trial, in which patients were receiving simvastatin, there was no adverse effect of Omacor on LDL cholesterol, which if anything decreased further. Fish oil treatment has also been associated with a deterioration in diabetic glycaemic control in some studies, ${ }^{14-18}$ but not in others ${ }^{19-22}$ and, as in non-diabetics, may increase LDL cholesterol. ${ }^{19}$ In the present trial, glycaemic control and LDL cholesterol were not adversely affected by Omacor in eight diabetic patients during one year of treatment or in 15 patients treated with Omacor for 24 weeks.

Increased fish oil intake achieved by either increased consumption of omega- 3 fats $^{8}$ or direct supplementation ${ }^{10}$ can prolong survival following myocardial infarction. There has been speculation about whether the effect might be caused by diminished serum triglycerides, decreased platelet aggregation or suppression of ventricular dysrhythmias. ${ }^{13}$ Serum triglyceride concentrations do appear to be a major determinant of survival in patients with coronary heart disease even after revascularisation. ${ }^{23}$ In GISSI, however, the dose of omega-3 fatty acids was equivalent to Omacor $1 \mathrm{~g}$ daily, ${ }^{10}$ which is one quarter of the dose used in the present study. Its effect on serum triglycerides, although significant, was small $(-3.4 \%$ with respect to baseline and $-4.8 \%$ compared to placebo). The patients in the GISSI trial were not selected for hypertriglyceridaemia and their average serum triglyceride value was $1.8 \mathrm{mmol} / 1$. Almost half also received statin treatment. It was uncertain whether the very small decrease in serum triglycerides in GISSI was caused by the low dose of omega- 3 fatty acids employed, an erosion of their effect on serum triglycerides due to the coadministration of statin treatment, or a lack of sustained effect. In the present study the dose of Omacor used ( $2 \mathrm{~g}$ twice daily) was chosen because it had previously been shown to lower serum triglyceride concentrations substantially in patients with hypertriglyceridaemia. ${ }^{11}$ The sustained decrease in serum triglycerides of 20-30\% despite statin treatment, which we now report, strongly suggests that the smaller effect in the GISSI study was caused by the lower dose of omega-3 fatty acids used. In that trial the decrease in coronary mortality was attributed to the anti-dysrhythmic properties of omega- 3 fatty acids. The question thus remains unanswered as to whether larger doses of omega-3 fatty acids with greater triglyceride lowering potential would be even more effective in reducing clinical end points, perhaps also affecting those related to atheroma progression.

Whatever the explanation for the decreased all cause mortality with fish oil, evidence for improved survival is lacking in the case of the more commonly prescribed class of triglyceride lowering agents, the fibrates, ${ }^{24}$ although in the most recent trial of gemfibrozil in myocardial infarction a $31 \%$ decrease in serum triglycerides was associated with a non-significant decrease in mortality of $11 \% .^{25}$ This result is, however, of limited relevance to the potential benefits of treating patients with hypertriglyceridaemia because the mean triglyceride concentration in the participants was only $1.6 \mathrm{mmol} / 1$. Furthermore the combination of fibrate with statin treatment has the potential to cause myositis, and fibrates, unlike the statins and fish oil, are contraindicated in renal impairment. ${ }^{24}$ Statins themselves have a triglyceride lowering effect, which, although variable, 
tends to be related to the degree of LDL reduction achieved. ${ }^{26}$ Many patients with combined hyperlipidaemia with relatively greater increases in triglycerides than cholesterol thus have persistent hypertriglyceridaemia even after the introduction of statin treatment. The present study shows that Omacor can, under these circumstances and in the doses we used, safely decrease serum triglycerides by a further $20-30 \%$ and that it is well tolerated, which has not been our previous experience with whole fish oil in doses required to achieve similar triglyceride lowering. The decision was made in designing this trial not to study patients on a standardised dose of simvastatin, but on the dose to which the patient had been titrated in the clinic. This design has the advantage that the method of arriving at the dose of simvastatin mirrors clinical practice and also that it allows us to conclude that the triglyceride response to Omacor is not influenced by the dose of simvastatin.

Given the strength of the evidence for the benefit of statin treatment in myocardial infarction survivors, ${ }^{1-3}$ it is probably unethical to consider further trials of statin versus placebo in patients with established CHD. However, the present trial shows that, with minimal safety monitoring, it would be possible to conduct a trial in patients already receiving statin treatment to establish whether omega-3 PUFA in the form of Omacor in the doses used in this study will confer additional benefit over the lower doses used in GISSI.

Support for this study was gratefully received from Pronova a.s. Norway. We are grateful to Ms C Price for expertly typing the manuscript.

1 Scandinavian Simvastatin Survival Study Group. Randomised trial of cholesterol lowering in 4444 patients with coronary heart disease; the Scandinavian survival study. Lancet 1994;344:1383-9.

2 The Long-Term Intervention with Pravastatin in Ischaemic Disease (LIPID) Study Group. Prevention of cardiovascular events and death with pravastatin in patients with cular events and death with pravastatin in patients with terol levels. N Engl f Med 1998;339:1349-57.

3 Sacks FM, Pfeffer MA, Moye LA, et al. The effect of pravastatin on coronary events after myocardial infarction in astatin on coronary events after myocardial infarction in patients with

4 Phillipson BE, Rothrock DW, Connor WE, et al. Reduction of plasma lipids, lipoproteins, and apoproteins by dietary fish oils in patients with hypertriglyceridemia. $N$ Engl F Med 1985;312:1210-6.

5 Contacos C, Barter PJ, Sullivan DR. Effect of pravastatin and omega-3 fatty acids on plasma lipids and lipoprotein in patients with combined hyperlipidemia. Arterioscler Thromb 1993;13:1755-62.
6 Nordoy A, Bonaa KH, Nilsen $\mathrm{H}$, et al. Effects of simvastatin and omega-3 fatty acids on plasma lipoprotein and lipid peroxidation in patients with combined hyperlipidaemia. $\mathcal{F}$ Int Med 1998;243:163-70.

7 Nakamura N, Hamazaki T, Ohta M, et al. Joint effects of HMG-CoA reductase inhibitors and eicosapentanoic acids on serum lipid profile and fatty acid concentrations in patients with hyperlipidaemia. Int F Clin Lab Res 1999;29: $22-5$.

8 Burr ML, Gilbert JF, Holliday RM, et al. Effects of changes in fat, fish and fibre intakes on death and myocardial reinfarction: diet and reinfarction trial (DART). Lancet 1989;ii:757-61.

9 De Lorgeril M, Salen P, Martin J-L, et al. Mediterranean diet, traditional risk factors, and the rate of cardiovascular complications after myocardial infarction: final report of the Lyon diet heart study. Circulation 1999;99:779-85.

10 GISSI-Prevenzione Investigators. Dietary supplementation with $\mathrm{n}-3$ polyunsaturated fatty acids and vitamin E Prevenzione trial. Lancet 1999;354:447-55.

11 Mackness MI, Bhatnagar D, Durrington PN, et al. Effect of a new fish oil concentrate on plasma lipids and lipoproteins in patients with hypertriglyceridaemia. Eur $\mathfrak{f}$ Clin Nutr in patients with

12 Harris WS. Fish oils and plasma lipid and lipoprotein metabolism in humans: a critical review. F Lipid Res 1989; 30:785-807.

13 Stone NJ. Fish consumption, fish oil, lipids and coronary heart disease. Am f Clin Nutr 1997;65:1083-6.

14 Friday KE, Childs MT, Tsunehara $\mathrm{CH}$. Elevated plasma glucose and lowered triglyceride levels from omega-3 fatty acid supplementation in type II diabetes. Diabetes Care 1987;12:276-81.

15 Glauber H, Wallace P, Griver K, et al. Adverse metabolic effect of omega-3 fatty acids in non-insulin-dependent diabetes mellitus. Ann Intern Med 1988;108:663-8.

16 Kasim SE. Dietary marine fish oils and insulin action in type 2 diabetes. Ann NY Acad Sci 1993;683:250-7.

17 Connor WE, Prince MJ, Ullmann D, et al. The hypotriglyceridaemic effect of fish oll Ann NY Acad Sci 1993;683:33740 .

18 Zambon S, Friday KE, Childs MT, et al. Effect of glyburide and omega 3 fatty acid dietary supplements on glucose and lipid metabolism in patients with non-insulin-dependent diabetes mellitus. Am 7 Clin Nutr 1992;56:447-54

19 Westerveld HT, de Graaf JC, van Breugel HH, et al. Effects of low-dose EPAE on glycemic control, lipid profile, lipoprotein (a), platelet aggregation, viscosity and platelet and vessel wall interaction in NIDDM. Diabetes Care 1993; 16:683-8.

20 Sirtori CR, Paoletti R, Mancini M, et al. N-3 fatty acids do not lead to an increased diabetic risk in patients with hyperlipidaemia and abnormal glucose tolerance. Italian fish oil multicenter-study. Am f Clin Nutr 1997;65:187481 .

21 Axelrod L, Camuso J, Williams E, et al. Effects of a small quantity of omega-3 fatty acids on cardiovascular risk factors in NIDDM: a randomised, prospective, double-blind, controlled study. Diabetes Care 1994;17:37-44.

22 Friedberg CE, Janssen MJ, Heine RJ, et al. Fish oil and glycemic control in diabetes. A meta-analysis. Diabetes Care 1998;21:494-500.

23 Linden T, Bondjers G, Karlsson T, et al. Serum triglycerides and HDL cholesterol-major predictors for long-term survival after coronary surgery. Eur Heart $\mathcal{f}$ 1994:15:74752.

24 Durrington PN, Illingworth R. Lipid-lowering drugs: who gets what? Current Opinion in Lipidology 1998;9:289-94.

25 Rubins HB, Robins SJ, Collins D, et al. Gemfibrozil for the secondary prevention of coronary heart disease in men with low levels of high-density lipoprotein cholesterol. $N$ Engl f Med 1999;341:410-8.

26 Stein EA, Lane M, Laskarzewski P. Comparisons of statins in hypertriglyceridaemia. Am f Cardiol 1998;81:66B-9B. 